\title{
Interaction effect among the different types of containers, used for storing seed, seed treatments and locations in jute variety $\mathrm{O}-9897$ on disease incidence, seed quality and yield
}

\begin{abstract}
The present study was carried out with the objective was to to identify suitable seed management practices that permit maximal fiber production. The experiments were conducted in the field of Jute Agriculture Experimental Station (JAES), Manikgonj and Kishoregonj Regional Station (KRS), Kishoregonj of BJRI. The experiments were conducted during the period April 2012 to January 2013. Six different types of containers viz. tin pot, plastic pot, poly bag, gunny bag lined with polyethylene, cloth bag, and the IRRI poly bag, two different seed treatments viz. Provax-200 and BAU- Biofungicide and two locations viz. JAES and KRS were used for the present study. The highest disease incidence $(10.86 \%)$ was encountered in case of interaction among plastic pot storing seeds and control condition. Interaction between different types of containers and seed treatments, the highest germination $(68.17 \%)$ was recorded in interaction effect of tin pot storing seeds and BAUBiofungicide treated seeds. The stick yield/ha (11.14ton) and fiber yield/ha (6.81ton) were recorded in interaction effect of plastic pot storing seeds and BAU- Biofungicide treated seeds. Highest seed yield/ ha $(422.44 \mathrm{~kg})$ was observed in the interaction of tin pot storing seeds and BAU- Biofungicide treated seeds. The highest disease incidence $(12.84 \%)$ was encountered in interaction effect of plastic pot storing seeds, control condition and KRS. Highest fiber yield/ha (6.81ton) and stick yield/ha (11.14ton) were recorded in interaction of plastic pot storing seeds, BAU- Biofungicide treated seeds and JAES. Highest seed yield/ ha $(422.44 \mathrm{~kg})$ was recorded in the interaction of tin pot storing seeds, BAU- Biofungicide treated seeds and JAES. So, the following recommendation may be drawn quality of jute seeds can be maintained by treatment with BAU Biofungicide and storage in tin pots.
\end{abstract}

Volume 2 Issue 5 - 2015

\section{SMA Haque}

Plant Pathology Department, Bangladesh Jute Research Institute, Bangladesh

Correspondence: SMA Haque, Plant Pathology Department, Bangladesh Jute Research Institute, Bangladesh, Email upolbjri@gmail.com

Received: April 08, 2015 | Published: October 06, 2015

Keywords: interaction, containers, seed treatments, locations, disease incidence, seed quality, yield, O-9897

\section{Abbreviations: B, BAU-Biofungicide; P, Provax $200 \mathrm{WP}$ \\ Introduction}

Jute is one of the major cash crops of Bangladesh. Its influence on ecology and economy is so intimate that it's the effects are significantly related to the Agro-ecology and the socioeconomic life of the people. Jute crop is also cultivated in different countries. The jute crop also greatly improves the soil fertility status by incorporating organic matter to the soil through decomposition of shaded leaves and plant residues and helps in breaking plough-pans through its long taproots. Also, jute and jute goods have been recognized as being friendly to the environment. Jute is mostly grown in the Indo-Bangladesh region and in some countries of Southeast Asia. Among the jute growing countries of the world, Bangladesh was second position in respect of production. ${ }^{1}$ The land and climatic conditions of Bangladesh are congenial for the production of high quality jute. In Bangladesh, about 0.709 million hectares of land were under jute cultivation and the total yield was 8.40 million bales. ${ }^{2,3}$ As per Khandakar, ${ }^{4}$ Bangladesh annually needs about 4000 metric tons of jute seeds of which only $12-15 \%$ is produced and supplied by the Bangladesh Agricultural Development Corporation (BADC). The rest of the seeds, about $85 \%$ or more of the requirements, are produced and managed by farmers'. ${ }^{5}$ Jute suffers from more than 13 different diseases, ${ }^{6}$ and 10 of them are seed borne. Sowing of infected seeds may cause the death of seedlings and often plants escaping early infection succumb to death due to different diseases. Seed germination decreases with the increase of the seed borne infection. The Seeds having higher seed borne infection results to significantly higher amount of disease development in the field. The rate of transmission of these pathogens from infected seeds for the growing plants and finally to the harvested seeds was relatively low. ${ }^{7}$ Among the seed-borne fungal diseases, stem-rot, black-band, and anthracnose caused by Macrophomina phaseolina (Tassi, Goid), Botryodiplodia theobromae and Colletotrichum corchori, ${ }^{8}$ respectively, are frequently transmitted through jute seeds. ${ }^{9-11}$ Macrophomina phaseolina alone can cause $10 \%$ yield loss. ${ }^{12}$ Stem rot, black band, anthracnose, foot rot and wilt (Rhizoctonia solani) and leaf mosaic virus are responsible for seed rot, pre and post emergence damping off seedlings, the spread of the diseases to standing crops and loss and deterioration of quality of fiber. ${ }^{10-14}$ Soft rot, foot rot and wilt caused by Sclerotium rolfsii and Rhizoctonia solani, respectively, also cause considerable yield losses to the crop. Cercospora leaf spot and target spot caused by Cercospora chorchori and Corynespora cassicola, respectively, are not so important, though these two pathogens are frequently transmitted through jute seeds. The pathogens like Fusarium spp. (Fusarium semitectum and Fusarium oxysporum), Curvularia lunata and Phomopsis ssp., are responsible for causing germination failure and seed rot. ${ }^{9}$ Yield loss due to seed borne diseases of jute is $8-20 \%$, 
depending on the severity of jute diseases from year to year. ${ }^{15}$ Infected jute seed fails to germinate or the young seedlings emerging from the infected seed die. Infection of jute seed causes germination failure, post emergence damping off and seedling blight. ${ }^{16}$ Jute seedlings or growing plants produced in the field from the infected seeds and escaping early infection may often be infected at the later stages of their growth by the primary seed borne inocula grown and multiplied on the infected dead seeds and seedlings. Seed borne pathogens causing diseases on the growing jute plants in the field quite often attack the capsules or pods and subsequently infect the seed, resulting in production of infected or unhealthy seeds. Therefore, proper disease control measures should be taken for the production of quality jute seeds. Considering the above facts, the present study was carried out with the objective was to find out suitable seed management for quality jute seeds and fiber production.

\section{Materials and methods}

\section{Experimental sites and period}

The experiments were conducted in the field of Jute Agriculture Experimental Station (JAES), Manikgonj and Kishoregonj Regional Station (KRS), Kishoregonj of BJRI. The experiments were conducted during the period April 2012 to January 2013.

\section{Varieties used}

Seed of O-9897 was selected for this study.

\section{Containers used}

For this experiment six different types of storage containers were used, viz. $\mathrm{T}_{1}=$ Tin pot, $\mathrm{T}_{2}=$ Plastic pot, $\mathrm{T}_{3}=$ Poly bag having $25 \mu \mathrm{m}$ thickness, $\mathrm{T}_{4}=$ Gunny bag lined with polyethylene, $\mathrm{T}_{5}=$ Cloth bag and $\mathrm{T}_{6}=\mathrm{IRRI}$ (International Rice Research Institute) Poly bag (Super Grain bag II Z) having $78 \mu \mathrm{m}$ thickness.

\section{Seed Management}

i. Seed treated with $\mathrm{P}(0.4 \%$ of seed weight $)$

ii. Seed treated with B @ 3\% of seed weight [] (Hossain, 2011b)

iii. Control (Untreated)

\section{Seed treated with Provax -200WP}

Seeds were treated with $\mathrm{P}(5,6$ - dihydro -2-Methyl-1, 4-oxathin-3carboxinilide, Group: Oxathin) @ $0.4 \%$ of seed weight in a $250 \mathrm{ml}$ Erlenmeyer flask and shaken thoroughly for proper coating of the seeds with the fungicides. ${ }^{17}$

\section{Seed treated with BAU- Biofungicide}

Seeds were treated with B @ 3\% of seed weight in a 250Ml Erlenmeyer flask and shaken thoroughly for proper coating of the seeds. The treated seeds were kept inside the brown paper bags so that seeds remain in dry condition till for further use.

\section{Experimental design}

The experiments were conducted following Randomized Block Design (RCBD) having three replications. The size of the unit plot was $10 \mathrm{~m}^{2}(5 \mathrm{mx} 2 \mathrm{~m})$ and the distance between plots and replications were $1.0 \mathrm{~m}$ and $1.0 \mathrm{~m}$, respectively.

\section{Soil characteristics and nutrient status}

The Soil characteristics and nutrient status of the two experimental stations (JAES, Manikgonj and KRS, Kishoregonj) are shown in Table 1.

\section{Application of fertilizers}

During final land preparation Urea 60kg, Triple Super Phosphate $50 \mathrm{~kg}$ and Muriate of Potash $25 \mathrm{~kg}$ per hectare were applied. ${ }^{18,19}$ After 15-20 days of seed germination first top dressing with the urea @ $60 \mathrm{~kg}$ and again another 15 days later of first top dressing, the $2^{\text {nd }}$ top dressing was given with $60 \mathrm{~kg}$ per hectare. Top dressing of urea was done very carefully so that it will not come in contact with the plant parts. To meet sulfur and zinc deficiency, gypsum and zinc oxide @ $45 \mathrm{~kg}$ and $5 \mathrm{~kg}$ per hectare were applied..$^{18,19}$

\section{Sowing of seeds}

Seeds were sown in line on 20 April, 2012 in Kishoregonj Regional Station (KRS), Kishoregonj and $2^{\text {nd }}$ May, 2012 in Jute Agriculture Experimental Station (JAES), Manikgonj. Row to row and plant to plant distance were maintained as $1 \mathrm{M}$ and $1 \mathrm{M}$, respectively. The seed rate for O-9897 was $4 \mathrm{~kg}$ per hectare.

\section{Data collection}

Data on different parameters were collected as shown below

a) Field emergence (germination \%)

b) Plant stand/ plant population

c) Incidence of diseases (\%)

d) Plant height $(\mathrm{M})$

e) Base diameter ( $\mathrm{mm})$

f) Fiber yield per plant (gm)

g) Fiber yield per hectare (ton)

h) Stick yield per plant (gm)

i) Stick yield per hectare (ton)

j) Average number of branch per plant

k) Average number of fruits per plant

1) Seed yield per plant (gm)

m) Seed yield per hectare $(\mathrm{kg})$

n) Weight of 1000- seeds (gm)

Some plots were kept un-harvested for seed production

\section{Statistical analysis}

Data were analyzed statistically and treatments effects were compared by Duncan's Multiple Range Test (DMRT). The relation between seed borne fungal pathogens and germination was observed with regression equations. Relationships between disease severity and 
seed, fiber and stick yield were also observed by linear regression lines and equations..$^{20}$

\section{Results}

\section{Interaction effect among the different types of containers used for storing seed and seed treatments on disease incidence, seed yield, stick yield and fiber}

\section{yield following line sowing method in the field}

Interaction effects of different seed treatments with different types of storage containers used for seed storing differed significantly for disease incidence, field emergence, a number of plant, plant height, base diameter, fiber yield, stick yield, number of branches, number of capsule, seed yield and 1000- seed weight (Tables 2\&3). In case of JAES, Interaction effect among Tin pot and seed treated with $\mathrm{B}$ resulted lower disease incidence $(2.95 \%)$. The highest disease incidence $(10.86 \%)$ was encountered in case of interaction among plastic pot and control condition.

Interaction effects between different types of containers and seed treatments on germination were found significant (Table 2). But there was no significant differences among $\mathrm{T}_{1} \mathrm{XD}(68.17 \%), \mathrm{T}_{2} \mathrm{XD}$ (64.33\%), $\mathrm{T}_{3} \mathrm{XD}_{1}(66.67 \%), \mathrm{T}_{4} \mathrm{XD}_{1}(65.17 \%), \mathrm{T}_{1} \mathrm{XD}_{2}(68.17 \%)$ and $\mathrm{T}_{3} \mathrm{XD}(66.67 \%)$. Again, there was no significant variation among $\mathrm{T}_{2} \mathrm{XD}_{1}(64.33 \%), \mathrm{T}_{4} \mathrm{XD}_{1}(65.17 \%), \mathrm{T}_{5} \mathrm{XD}_{1}(61.17 \%), \mathrm{T}_{6} \mathrm{XD}_{1}(59.67 \%)$, $\mathrm{T}_{2} \mathrm{XD}_{2}(62.33 \%), \mathrm{T}_{4} \mathrm{XD}_{2}(61.00 \%), \mathrm{T}_{4} \mathrm{XD}_{2}(61.00 \%), \mathrm{T}_{3} \mathrm{XD}_{3}(57.67 \%)$ and $\mathrm{T}_{6} \mathrm{XD}(55.83 \%), \mathrm{T}_{1} \mathrm{XD}_{3}(58.00 \%), \mathrm{T}_{2} \mathrm{XD}_{3}(55.50 \%), \mathrm{T}_{3} \mathrm{XD}_{3}$ (57.67\%), $\mathrm{T}_{4} \mathrm{XD}_{3}(54.50 \%), \mathrm{T}_{5} \mathrm{XD}_{3}(53.83 \%), \mathrm{T}_{6} \mathrm{XD}_{3}(57.67 \%)$. The highest result was found in $\mathrm{T}_{1} \mathrm{XD}_{1}$ and $\mathrm{T}_{3} \mathrm{XD}_{1}(68.17 \%)$ followed by $\mathrm{T}_{3} \mathrm{XD}_{2}(66.67 \%)$. The lowest result was found in $\mathrm{T}_{5} \mathrm{XD}_{3}(53.83 \%)$ preceded by $\mathrm{T}_{4} \mathrm{XD}_{3}(54.50 \%)$.

Interaction effects between different types of containers and seed treatments on a plant stand $/ \mathrm{m}^{2}$ were found significant. But there were no significant differences among $\mathrm{T}_{6} \mathrm{XD}_{2}, \mathrm{~T}_{1} \mathrm{XD}_{3}(26.60)$ and $\mathrm{T}_{2} \mathrm{XD}_{3} \mathrm{~T}_{6} \mathrm{XD}_{3}$ (23.33) The highest result was found in $\mathrm{T}_{1} \mathrm{XD}_{1}(40.60)$ followed by $\mathrm{T}_{5} \mathrm{XD}_{1}$ (37.33). The lowest result was found in $\mathrm{T}_{3} \mathrm{XD}_{3}$ (21.93) preceded by $\mathrm{T}_{5} \mathrm{XD}_{3}(22.40)$.

Interaction effects between different types of containers and seed treatments on plant height were found no significance. The highest result was found in $\mathrm{T}_{2} \mathrm{XD} \mathrm{D}_{1}(3.17 \mathrm{M})$ followed by $\mathrm{T}_{4} \mathrm{XD_{1 }}(3.11 \mathrm{M})$. The lowest result was found in $\mathrm{T}_{5} \mathrm{XD}_{3}(2.75 \mathrm{M})$ preceded by $\mathrm{T}_{3} \mathrm{XD}$ $(2.76 \mathrm{M})$. In cases of base diameter there was no significant differences among $\mathrm{T}_{1} \mathrm{XD}(16.14 \mathrm{~mm}), \mathrm{T}_{2} \mathrm{XD}_{1}(16.69 \mathrm{~mm}), \mathrm{T}_{3} \mathrm{XD}_{1}(16.51 \mathrm{~mm})$, $\mathrm{T}_{4} \mathrm{XD}_{1}(15.81 \mathrm{~mm}), \mathrm{T}_{5} \mathrm{XD}_{1}(16.39 \mathrm{~mm}), \mathrm{T}_{6} \mathrm{XD}_{1}(16.31 \mathrm{~mm}), \mathrm{T}_{1} \mathrm{XD}$ (16.05mm), $\mathrm{T}_{2} \mathrm{XD}_{2}(15.75 \mathrm{~mm}), \mathrm{T}_{3} \mathrm{XD}_{2}(15.76 \mathrm{~mm}), \mathrm{T}_{5} \mathrm{XD}_{2}(15.59 \mathrm{~mm})$, $\mathrm{T}_{6} \mathrm{XD}_{2}(15.51 \mathrm{~mm}), \mathrm{T}_{1} \mathrm{XD}_{3}(15.70 \mathrm{~mm}), \mathrm{T}_{2} \mathrm{XD}_{3}(15.34 \mathrm{~mm})$ and $\mathrm{T}_{3} \mathrm{XD}_{3}$ $(15.46 \mathrm{~mm})$. Again, there was no significant variation among $\mathrm{T}_{2} \mathrm{XD}_{2}$ (15.75mm), $\mathrm{T}_{3} \mathrm{XD}_{2}(15.76 \mathrm{~mm}), \mathrm{T}_{4} \mathrm{XD}_{2}(15.13 \mathrm{~mm}), \mathrm{T}_{5} \mathrm{XD}_{2}(15.59 \mathrm{~mm})$, $\mathrm{T}_{6} \mathrm{XD}_{2}(15.51 \mathrm{~mm}), \mathrm{T}_{1} \mathrm{XD}_{3}(15.70 \mathrm{~mm}), \mathrm{T}_{2} \mathrm{XD}_{3}(15.34 \mathrm{~mm}), \mathrm{T}_{3} \mathrm{XD}_{3}$ $(15.46 \mathrm{~mm}), \mathrm{T}_{4} \mathrm{XD}_{3}(15.06 \mathrm{~mm})$ and $\mathrm{T}_{5} \mathrm{XD}_{3}(15.03 \mathrm{~mm})$, the highest result was found in $\mathrm{T}_{2} \mathrm{XD}_{1}(16.69 \mathrm{~mm})$ and lowest result was found in $\mathrm{T}_{6} \mathrm{XD}_{3}(14.55 \mathrm{~mm})$.

Interaction effects between different types of containers and seed treatments on fiber yield/ha were found significant. But there was no significant differences among $\mathrm{T}_{1} \mathrm{XD}_{1}$ (5.64ton), $\mathrm{T}_{2} \mathrm{XD}_{1}$ (6.81ton), $\mathrm{T}_{5} \mathrm{XD}_{1}\left(6.21\right.$ ton) and $\mathrm{T}_{6} \mathrm{XD}_{1}$ (6.07ton). Again, there was no significant variation among $\mathrm{T}_{4} \mathrm{XD}$ (3.69ton), $\mathrm{T}_{1} \mathrm{XD}_{2}$ (2.66ton), $\mathrm{T}_{2} \mathrm{XD}_{2}$ (3.36ton), $\mathrm{T}_{3} \mathrm{XD}_{2}$ (3.59ton), $\mathrm{T}_{4} \mathrm{XD}_{2}$ (3.17ton), $\mathrm{T}_{5} \mathrm{XD}_{2}$ (3.69ton), $\mathrm{T}_{1} \mathrm{XD}_{3}$ (3.08ton),
$\mathrm{T}_{2} \mathrm{XD}_{3}$ (2.33ton), $\mathrm{T}_{3} \mathrm{XD}_{3}$ (2.19ton), $\mathrm{T}_{4} \mathrm{XD}_{3}$ (2.80ton), $\mathrm{T}_{5} \mathrm{XD}_{3}$ (3.08ton) and $\mathrm{T}_{6} \mathrm{XD}_{3}$ (2.66ton). The highest result was found in $\mathrm{T}_{2} \mathrm{XD}_{1}$ (6.81ton) followed by $\mathrm{T}_{5} \mathrm{XD}_{1}$ (6.21ton). The lowest result was found in $\mathrm{T}_{3} \mathrm{XD}_{3}$ (2.19ton) preceded by $\mathrm{T}_{2} \mathrm{XD}_{3}$ (2.33ton).

Interaction effects between different types of containers and seed treatments on stick yield/ha were found significant. But there was no significant differences among $\mathrm{T}_{1} \mathrm{XD}_{1}$ (10.76ton), $\mathrm{T}_{2} \mathrm{XD}_{1}$ (11.14ton) and $\mathrm{T}_{5} \mathrm{XD}_{1}$ (11.04ton). Again, there was no significant variation among $\mathrm{T}_{1} \mathrm{XD}_{3}$ (5.58ton), $\mathrm{T}_{2} \mathrm{XD}_{3}$ (6.14ton), $\mathrm{T}_{3} \mathrm{XD}_{3}$ (5.05ton), $\mathrm{T}_{4} \mathrm{XD}_{3}$ (4.95ton) and $\mathrm{T}_{5} \mathrm{XD}_{3}$ (5.63ton). The highest result was found in $\mathrm{T}_{2} \mathrm{XD}_{1}$ (11.14ton) followed by $\mathrm{T}_{5} \mathrm{XD}_{1}$ (11.04ton). The lowest result was found in $\mathrm{T}_{4} \mathrm{XD}_{3}$ (4.95ton) preceded by $\mathrm{T}_{2} \mathrm{XD}_{3}$ (5.05ton).

Interaction effects between different types of containers and seed treatments on seed yield/ha were found significant. But there was no significant differences among $\mathrm{T}_{1} \mathrm{XD}_{1}(422.44 \mathrm{~kg})$ and $\mathrm{T}_{6} \mathrm{XD}_{1}$ $(403.12 \mathrm{~kg})$. Again, there was no significant variation among $\mathrm{T}_{2} \mathrm{XD}_{1}$ (356.32kg), $\mathrm{T}_{5} \mathrm{XD}_{1}(344.65 \mathrm{~kg}), \mathrm{T}_{2} \mathrm{XD}_{2}(327.67 \mathrm{~kg}), \mathrm{T}_{3} \mathrm{XD}_{2}(356.23 \mathrm{~kg})$, $\mathrm{T}_{5} \mathrm{XD}_{2}(335.66 \mathrm{~kg}), \mathrm{T}_{6} \mathrm{XD}_{2}(342.46 \mathrm{~kg}), \mathrm{T}_{1} \mathrm{XD}_{3}(348.75 \mathrm{~kg}), \mathrm{T}_{5} \mathrm{XD}_{3}$ $(334.25 \mathrm{~kg}), \mathrm{T}_{6} \mathrm{XD}_{3}(337.56 \mathrm{~kg})$ and $\mathrm{T}_{2} \mathrm{XD}_{2}(327.67 \mathrm{~kg}), \mathrm{T}_{4} \mathrm{XD}_{2}$ (323.43kg), $\mathrm{T}_{2} \mathrm{XD}_{3}(314.34 \mathrm{~kg}), \mathrm{T}_{3} \mathrm{XD}_{3}(304.55 \mathrm{~kg}), \mathrm{T}_{4} \mathrm{XD}_{3}(313.65 \mathrm{~kg})$. The highest result was found in $\mathrm{T}_{1} \mathrm{XD}_{1}(422.44 \mathrm{~kg})$ followed by $\mathrm{T}_{6} \mathrm{XD}_{1}(403.12 \mathrm{~kg})$. The lowest result was found in $\mathrm{T}_{3} \mathrm{XD}_{3}(304.55 \mathrm{~kg})$ preceded by $\mathrm{T}_{4} \mathrm{XD}_{3}(313.65 \mathrm{~kg})$.

Interaction effects between different types of containers and seed treatments on 1000-seed weight were found significant. $\mathrm{T}_{1} \mathrm{XD}_{1}$ (1.49gm), $\mathrm{T}_{2} \mathrm{XD}_{1}(1.67 \mathrm{gm}), \mathrm{T}_{3} \mathrm{XD}_{1}(1.84 \mathrm{gm}), \mathrm{T}_{4} \mathrm{XD}_{1}(1.25 \mathrm{gm}), \mathrm{T}_{5} \mathrm{XD}_{1}$ (1.56gm), $\mathrm{T}_{6} \mathrm{XD}_{1}(1.22 \mathrm{gm}), \mathrm{T}_{1} \mathrm{XD}_{2}(1.45 \mathrm{gm}), \mathrm{T}_{2} \mathrm{XD}_{2}(1.75 \mathrm{gm}), \mathrm{T}_{3} \mathrm{XD}_{2}$ $(1.67 \mathrm{gm}), \mathrm{T}_{4} \mathrm{XD}_{2}(1.25 \mathrm{gm}), \mathrm{T}_{5} \mathrm{XD}_{2}(1.55 \mathrm{~mm}), \mathrm{T}_{1} \mathrm{XD}_{3}(1.45 \mathrm{~mm})$ and $\mathrm{T}_{2} \mathrm{XD}_{3}(1.68 \mathrm{~mm})$. The highest result was found in $\mathrm{T}_{3} \mathrm{XD}_{1}(1.84 \mathrm{gm})$ followed by $\mathrm{T}_{2} \mathrm{XD}_{2}(1.75 \mathrm{gm})$. The lowest result was found in $\mathrm{T}_{5} \mathrm{XD}_{3}$ $(1.12 \mathrm{gm})$ preceded by $\mathrm{T}_{3} \mathrm{XD}_{3}(1.15 \mathrm{gm})$ (Table 2$)$. In case of KRS, Interaction effect among Tin pot and seed treated with $\mathrm{B}$ resulted lower disease incidence $(2.35 \%)$. The highest disease incidence $(12.84 \%)$ was encountered in case of interaction among plastic pot and control condition.

Interaction effects between different types of containers and seed treatments on germination were found significant (Table 3). But there was no significant differences among $\mathrm{T}_{1} \mathrm{XD}_{1}(68.00 \%), \mathrm{T}_{3} \mathrm{XD}_{1}$ (65.50\%), $\mathrm{T}_{4} \mathrm{XD}_{1}(65.67 \%), \mathrm{T}_{1} \mathrm{XD}_{2}(68.00 \%)$ and $\mathrm{T}_{3} \mathrm{XD}_{2}(63.83 \%)$. Again, there was no significant variation among $\mathrm{T}_{6} \mathrm{XD}_{2}(59.67 \%)$, $\mathrm{T}_{2} \mathrm{XD}_{3}(56.83 \%), \mathrm{T}_{3} \mathrm{XD}_{3}(58.50 \%), \mathrm{T}_{4} \mathrm{XD}_{3}(55.00 \%)$ and $\mathrm{T}_{6} \mathrm{XD}_{3}$ $(59.33 \%)$. The highest result was found in $\mathrm{T}_{1} \mathrm{XD}_{1}$ and $\mathrm{T}_{1} \mathrm{XD}_{2}(68.00 \%)$ followed by $\mathrm{T}_{4} \mathrm{XD}(65.67 \%)$. The lowest result was found in $\mathrm{T}_{4} \mathrm{XD}_{3}$ $(55.00 \%)$ preceded by $\mathrm{T}_{2} \mathrm{XD}_{3}(56.83 \%)$.

Interaction effects between different types of containers and seed treatments on a plant stand $/ \mathrm{m}^{2}$ were found significance. But there were no significant differences among $\mathrm{T}_{5} \mathrm{XD}_{1}(25.30), \mathrm{T}_{1} \mathrm{XD}_{2}$ (24.93), $\mathrm{T}_{2} \mathrm{XD}_{2}$ (24.20), $\mathrm{T}_{3} \mathrm{XD}_{2}$ (24.20), $\mathrm{T}_{4} \mathrm{XD}_{2}$ (23.10) and $\mathrm{T}_{3} \mathrm{XD}_{3}$ (23.10) The highest result was found in $\mathrm{T}_{1} \mathrm{XD}_{1}(34.83)$ followed by $\mathrm{T}_{6} \mathrm{XD}_{1}(31.53)$. The lowest result was found in $\mathrm{T}_{6} \mathrm{XD}_{3}(17.23)$ preceded by $\mathrm{T}_{5} \mathrm{XD}_{2}$ (17.60).

Interaction effects between different types of containers and seed treatments on plant height were found significant. But there was no significant differences among $\mathrm{T}_{1} \mathrm{XD}_{1}(3.14 \mathrm{M}), \mathrm{T}_{2} \mathrm{XD}_{1}(3.08 \mathrm{M})$, $\mathrm{T}_{3} \mathrm{XD}_{1}(2.89 \mathrm{M}), \mathrm{T}_{4} \mathrm{XD}_{1}(2.89 \mathrm{M}), \mathrm{T}_{5} \mathrm{XD}_{1}(2.78 \mathrm{M}), \mathrm{T}_{6} \mathrm{XD}_{1}(2.91 \mathrm{M})$ $\mathrm{T}_{3} \mathrm{XD}_{2}(2.79 \mathrm{M}), \mathrm{T}_{4} \mathrm{XD}_{2}(2.86 \mathrm{M}), \mathrm{T}_{5} \mathrm{XD}_{2}(2.83 \mathrm{M}), \mathrm{T}_{6} \mathrm{XD}_{2}(2.82 \mathrm{M})$ and $\mathrm{T}_{4} \mathrm{XD}(2.77 \mathrm{M})$. Again, there was no significant variation among 
$\mathrm{T}_{2} \mathrm{XD}_{2}(2.73 \mathrm{M}), \mathrm{T}_{3} \mathrm{XD}_{3}(2.73 \mathrm{M}), \mathrm{T}_{5} \mathrm{XD}_{3}(2.76 \mathrm{M}), \mathrm{T}_{6} \mathrm{XD}_{3}(2.76 \mathrm{M})$ and $\mathrm{T}_{1} \mathrm{XD}_{2}(2.69 \mathrm{M}), \mathrm{T}_{1} \mathrm{XD}_{3}(2.65 \mathrm{M}), \mathrm{T}_{2} \mathrm{XD}_{3}(2.67 \mathrm{M})$. The highest result was found in $\mathrm{T}_{1} \mathrm{XD}_{1}(3.14 \mathrm{M})$ followed by $\mathrm{T}_{2} \mathrm{XD}_{1}(3.08 \mathrm{M})$. The lowest result was found in $\mathrm{T}_{1} \mathrm{XD}_{3}(2.65 \mathrm{M})$ preceded by $\mathrm{T}_{2} \mathrm{XD}_{3}(2.67 \mathrm{M})$. In cases of base diameter there was no significant differences among $\mathrm{T}_{1} \mathrm{XD}_{1}(15.47 \mathrm{~mm}), \mathrm{T}_{2} \mathrm{XD}_{1}(15.23 \mathrm{~mm}), \mathrm{T}_{4} \mathrm{XD}_{1}(15.89 \mathrm{~mm}), \mathrm{T}_{5} \mathrm{XD}_{1}$ $(15.65 \mathrm{~mm}), \quad \mathrm{T}_{1} \mathrm{XD}_{2}(15.47 \mathrm{~mm}), \mathrm{T}_{4} \mathrm{XD}_{3}(15.31 \mathrm{~mm})$ and $\mathrm{T}_{6} \mathrm{XD}_{3}$ $(14.35 \mathrm{~mm})$. Again, there was no significant variation among $\mathrm{T}_{1} \mathrm{XD}_{2}$ (13.93mm), $\mathrm{T}_{2} \mathrm{XD}_{2}(14.18 \mathrm{~mm}), \mathrm{T}_{3} \mathrm{XD}_{2}(14.25 \mathrm{~mm}), \mathrm{T}_{1} \mathrm{XD}_{3}(14.45 \mathrm{~mm})$, $\mathrm{T}_{2} \mathrm{XD}_{3}(13.95 \mathrm{~mm}), \mathrm{T}_{1} \mathrm{XD}_{3}(14.23 \mathrm{~mm})$ and $\mathrm{T}_{5} \mathrm{XD}_{3}(13.76 \mathrm{~mm})$. The highest result was found in $\mathrm{T}_{6} \mathrm{XD}_{1}(19.57 \mathrm{~mm})$ and lowest result was found in $\mathrm{T}_{5} \mathrm{XD}_{3}(13.76 \mathrm{~mm})$.

Interaction effects between different types of containers and seed treatments on fiber yield/ha were found significant. But there was no significant differences among $\mathrm{T}_{1} \mathrm{XD}_{1}$ (3.55ton), $\mathrm{T}_{2} \mathrm{XD}$ (3.06ton), $\mathrm{T}_{3} \mathrm{XD}_{1}$ (3.85ton), $\mathrm{T}_{4} \mathrm{XD}_{1}$ (3.09ton), $\mathrm{T}_{5} \mathrm{XD}$ (3.32ton), $\mathrm{T}_{6} \mathrm{XD}$ (3.36ton), $\mathrm{T}_{1} \mathrm{XD}_{2}$ (3.50ton), $\mathrm{T}_{2} \mathrm{XD}_{2}$ (2.88ton), $\mathrm{T}_{3} \mathrm{XD}_{2}$ (2.67ton), $\mathrm{T}_{4} \mathrm{XD}_{2}$ (3.45ton), $\mathrm{T}_{5} \mathrm{XD}_{2}$ (3.44ton), $\mathrm{T}_{6} \mathrm{XD}_{2}$ (3.77ton), $\mathrm{T}_{1} \mathrm{XD}_{3}$ (2.48ton), $\mathrm{T}_{2} \mathrm{XD}_{3}$ (2.02ton), $\mathrm{T}_{3} \mathrm{XD}_{3}(2.57$ ton $)$ and $\mathrm{T}_{4} \mathrm{XD}_{3}$ (3.16ton). Again, there was no significant variation among $\mathrm{T}_{5} \mathrm{XD}_{3}$ (1.90ton) and $\mathrm{T}_{6} \mathrm{XD}_{3}$ (1.39ton). The highest result was found in $\mathrm{T}_{3} \mathrm{XD}$ (3.85ton) followed by $\mathrm{T}_{6} \mathrm{XD}$ (3.77ton). The lowest result was found in $\mathrm{T}_{6} \mathrm{XD}_{3}$ (1.39ton) preceded by $\mathrm{T}_{5} \mathrm{XD}_{3}$ (1.90ton).

Interaction effects between different types of containers and seed treatments on stick yield/ha were found significant. But there was no significant differences among $\mathrm{T}_{1} \mathrm{XD}_{1}$ (9.76ton) and $\mathrm{T}_{6} \mathrm{XD}_{1}$ (9.67ton). Again, there was no significant variation among $\mathrm{T}_{4} \mathrm{XD}_{1}$ (6.88ton), $\mathrm{T}_{5} \mathrm{XD}_{1}$ (7.04ton), $\mathrm{T}_{3} \mathrm{XD}_{2}$ (6.91ton), $\mathrm{T}_{4} \mathrm{XD}_{2}$ (6.62ton) and $\mathrm{T}_{1} \mathrm{XD}_{3}$ (5.18ton), $\mathrm{T}_{4} \mathrm{XD}_{3}$ (5.14ton), $\mathrm{T}_{5} \mathrm{XD}_{3}$ (4.63ton), $\mathrm{T}_{6} \mathrm{XD}_{3}$ (4.91ton). The highest result was found in $\mathrm{T}_{1} \mathrm{XD}_{1}$ (9.76ton) followed by $\mathrm{T}_{6} \mathrm{XD}$ (9.67ton). The lowest result was found in $\mathrm{T}_{3} \mathrm{XD}_{3}$ (4.33ton) preceded by $\mathrm{T}_{5} \mathrm{XD}_{3}$ (4.63ton).

Interaction effects between different types of containers and seed treatments on seed yield/ha were found significant. But there was no significant differences among $\mathrm{T}_{1} \mathrm{XD}_{1}(418.55 \mathrm{~kg}), \mathrm{T}_{6} \mathrm{XD}_{1}(403.12 \mathrm{~kg})$ and $\mathrm{T}_{1} \mathrm{XD}_{2}(406.34 \mathrm{~kg})$. Again, there was no significant variation among $\mathrm{T}_{3} \mathrm{XD}_{2}(322.55 \mathrm{~kg}), \mathrm{T}_{4} \mathrm{XD}_{2}(323.43 \mathrm{~kg})$ and $\mathrm{T}_{5} \mathrm{XD}_{3}(324.65 \mathrm{~kg})$. The highest result was found in $\mathrm{T}_{1} \mathrm{XD}(418.55 \mathrm{~kg})$ followed by $\mathrm{T}_{1} \mathrm{XD}_{2}(406.34 \mathrm{~kg})$. The lowest result was found in $\mathrm{T}_{4} \mathrm{XD}_{3}(313.65 \mathrm{~kg})$ preceded by $\mathrm{T}_{3} \mathrm{XD}_{2}(322.55 \mathrm{~kg})$.

Interaction effects between different types of containers and seed treatments on 1000-seed weight were found significant. But there was no significant differences among $\mathrm{T}_{1} \mathrm{XD}_{1}(1.39 \mathrm{gm}), \mathrm{T}_{2} \mathrm{XD}_{1}(1.85 \mathrm{gm})$, $\mathrm{T}_{3} \mathrm{XD}_{1}(1.78 \mathrm{gm}), \mathrm{T}_{5} \mathrm{XD}_{1}(1.53 \mathrm{gm}), \mathrm{T}_{2} \mathrm{XD}_{2}$ (1.65gm), $\mathrm{T}_{4} \mathrm{XD}_{2}(1.42 \mathrm{gm})$ and $\mathrm{T}_{1} \mathrm{XD}_{3}(1.39 \mathrm{gm})$. Again, there was no significant variation among $\mathrm{T}_{4} \mathrm{XD}_{1}(1.22 \mathrm{gm}), \mathrm{T}_{3} \mathrm{XD}_{3}(1.18 \mathrm{gm}), \mathrm{T}_{4} \mathrm{XD}_{3}(1.22 \mathrm{gm}), \mathrm{T}_{5} \mathrm{XD}_{3}(1.23 \mathrm{gm})$ and $\mathrm{T}_{6} \mathrm{XD}_{3}(1.22 \mathrm{gm})$. The highest result was found in $\mathrm{T}_{2} \mathrm{XD}_{1}(1.85 \mathrm{gm})$ followed by $\mathrm{T}_{3} \mathrm{XD}_{1}(1.78 \mathrm{gm})$. The lowest result was found in $\mathrm{T}_{3} \mathrm{XD}_{2}$ (1.11gm) preceded by $\mathrm{T}_{6} \mathrm{XD}_{1}$ and $\mathrm{T}_{6} \mathrm{XD}_{2}(1.12 \mathrm{gm})$ (Table 3$)$.

\section{Interaction effect among the different types of containers used for storing seed, seed treatments and locations on disease incidence, seed yield, stick yield and fiber yield following line sowing method in the} field

Interaction effect of locations, different seed treatments with different types of storage containers differed significantly for disease incidence, field emergence, number of plants, plant height, base diameter, fiber yield, stick yield, number of branches, number of capsules, seed yield and 1000- seed weight (Table 4). Interaction effect among Tin pot storing seeds, seed treated with B and KRS resulted lower disease incidence $(2.35 \%)$. The highest disease incidence $(12.84 \%)$ was encountered in case of interaction among plastic pot storing seeds, control condition and KRS.

Interaction effects between locations and different types of containers and seed treatments on germination were found significant. But there were no significant differences among $\mathrm{T}_{1} \mathrm{XD}_{1} \mathrm{XL}_{1}(68.17 \%)$, $\mathrm{T}_{2} \mathrm{XD}_{1} \mathrm{XL}_{1}$ (64.33\%), $\mathrm{T}_{3} \mathrm{XD}_{1} \mathrm{XL}_{1} \quad(66.67 \%), \mathrm{T}_{4} \mathrm{XD}_{1} \mathrm{XL}_{1} \quad(65.17 \%)$, $\mathrm{T}_{1} \mathrm{XD}_{2} \mathrm{XL}_{1}(68.17 \%), \mathrm{T}_{3} \mathrm{XD}_{2} \mathrm{XL}_{1}(66.67 \%), \mathrm{T}_{1} \mathrm{XD}_{1} \mathrm{XL}_{2}(68.00 \%)$, $\mathrm{T}_{3} \mathrm{XD}_{1} \mathrm{XL}_{2}(65.50 \%), \mathrm{T}_{4} \mathrm{XD}_{1} \mathrm{XL}_{2}(65.67 \%)$ and $\mathrm{T}_{1} \mathrm{XD}_{2} \mathrm{XL}_{2}(68.00 \%)$. Again, there was no significant variation among $\mathrm{T}_{2} \mathrm{XD}_{1} \mathrm{XL}_{1}(64.33 \%)$, $\mathrm{T}_{5} \mathrm{XD}_{1} \mathrm{XL}_{1}(61.17 \%), \mathrm{T}_{6} \mathrm{XD}_{1} \mathrm{XL}_{1}(59.67 \%), \mathrm{T}_{2} \mathrm{XD}_{2} \mathrm{XL}_{1}(62.33 \%)$, $\mathrm{T}_{4} \mathrm{XD}_{2} \mathrm{XL}_{1}(61.00 \%), \mathrm{T}_{5} \mathrm{XD}_{2} \mathrm{XL}_{1}(61.00 \%), \mathrm{T}_{5} \mathrm{XD}_{1} \mathrm{XL}_{2}(60.83 \%)$, $\mathrm{T}_{2} \mathrm{XD}_{2} \mathrm{XL}_{2}(62.17 \%), \mathrm{T}_{3} \mathrm{XD}_{2} \mathrm{XL}_{2}(63.83 \%), \mathrm{T}_{4} \mathrm{XD}_{2} \mathrm{XL}_{2}(61.83 \%)$ and $\mathrm{T}_{5} \mathrm{XD}_{2} \mathrm{XL}_{2}(61.83 \%)$. The highest result was found in $\mathrm{T}_{1} \mathrm{XD}_{1} \mathrm{XL}_{1}$ $(68.17 \%)$ followed by $\mathrm{T}_{1} \mathrm{XD}_{1} \mathrm{XL}_{2}$ and $\mathrm{T}_{1} \mathrm{XD}_{2} \mathrm{XL}_{2}(68.00 \%)$. The lowest result was found in $\mathrm{T}_{5} \mathrm{XD}_{3} \mathrm{XL}_{1}(53.83 \%)$ preceded by $\mathrm{T}_{4} \mathrm{XD}_{3} \mathrm{XL}_{1}(54.50 \%)$.

Interaction effects between locations and different types of containers and seed treatments on plant stand $/ \mathrm{m}^{2}$ were found significant. But there was no significant differences among $\mathrm{T}_{2} \mathrm{XD}_{1} \mathrm{XL}_{2}$ (25.67), $\mathrm{T}_{5} \mathrm{XD}_{1} \mathrm{XL}_{2}$ (25.30), $\mathrm{T}_{1} \mathrm{XD}_{2} \mathrm{XL}_{2}$ (24.93), $\mathrm{T}_{6} \mathrm{XD}_{2} \mathrm{XL}_{2}(25.30)$ and $\mathrm{T}_{2} \mathrm{XD}_{3} \mathrm{XL}_{1}(23.33), \mathrm{T}_{6} \mathrm{XD}_{3} \mathrm{XL}_{1}(23.33), \mathrm{T}_{4} \mathrm{XD}_{2} \mathrm{XL}_{2}(23.10), \mathrm{T}_{3} \mathrm{XD}_{3} \mathrm{XL}_{2}$ (23.10) The highest result was found in $\mathrm{T}_{1} \mathrm{XD}_{1} \mathrm{XL}_{1}(40.50)$ followed by $\mathrm{T}_{5} \mathrm{XD}_{1} \mathrm{XL}_{1}$ (37.33). The lowest result was found in $\mathrm{T}_{5} \mathrm{XD}_{3} \mathrm{XL}_{2}(16.50)$ preceded by $\mathrm{T}_{6} \mathrm{XD}_{3} \mathrm{XL}_{2}$ (17.23). Interaction effect between locations and different types of containers and seed treatments on plant height were found no significant. The highest result was found in $\mathrm{T}_{2} \mathrm{XD}_{1} \mathrm{XL}_{1}$ (3.17M) followed by $\mathrm{T}_{4} \mathrm{XD}_{1} \mathrm{XL}_{1}(3.11 \mathrm{M})$. The lowest result was found in $\mathrm{T}_{1} \mathrm{XD}_{3} \mathrm{XL}_{2}(2.65 \mathrm{M})$ preceded by $\mathrm{T}_{2} \mathrm{XD}_{3} \mathrm{XL}_{2}(2.67 \mathrm{M})$.

In cases of base diameter there was no significant differences among $\mathrm{T}_{1} \mathrm{XD}_{1} \mathrm{XL}_{1}(16.14 \mathrm{~mm}), \mathrm{T}_{2} \mathrm{XD}_{1} \mathrm{XL}_{1}(16.69 \mathrm{~mm}), \mathrm{T}_{3} \mathrm{XD}_{1} \mathrm{XL}_{1}(16.51 \mathrm{~mm})$, $\mathrm{T}_{4} \mathrm{XD}_{1} \mathrm{XL}_{1}(15.81 \mathrm{~mm}), \mathrm{T}_{5} \mathrm{XD}_{1} \mathrm{XL}_{1}(16.39 \mathrm{~mm}), \mathrm{T}_{6} \mathrm{XD}_{1} \mathrm{XL}_{1}(16.31 \mathrm{~mm})$, $\mathrm{T}_{1} \mathrm{XD}_{2} \mathrm{XL}_{1}(16.05 \mathrm{~mm}), \mathrm{T}_{2} \mathrm{XD}_{2} \mathrm{XL}_{1}(15.75 \mathrm{~mm}), \mathrm{T}_{3} \mathrm{XD}_{2} \mathrm{XL}_{1}(15.76 \mathrm{~mm})$, $\mathrm{T}_{4} \mathrm{XD}_{2} \mathrm{XL}_{1}(15.13 \mathrm{~mm}), \mathrm{T}_{5} \mathrm{XD}_{2} \mathrm{XL}_{1}(15.59 \mathrm{~mm}), \mathrm{T}_{6} \mathrm{XD}_{2} \mathrm{XL}_{1}(15.51 \mathrm{~mm})$, $\mathrm{T}_{1} \mathrm{XD}_{3} \mathrm{XL}_{1}(15.70 \mathrm{~mm}), \mathrm{T}_{3} \mathrm{XD}_{3} \mathrm{XL}_{1}(15.46 \mathrm{~mm}), \mathrm{T}_{4} \mathrm{XD}_{3} \mathrm{XL}_{1}(15.06 \mathrm{~mm})$, $\mathrm{T}_{1} \mathrm{XD}_{1} \mathrm{XL}_{2}(15.47 \mathrm{~mm}), \mathrm{T}_{3} \mathrm{XD}_{1} \mathrm{XL}_{2}(16.10 \mathrm{~mm}), \mathrm{T}_{4} \mathrm{XD}_{1} \mathrm{XL}_{2}(15.89 \mathrm{~mm})$, $\mathrm{T}_{5} \mathrm{XD}_{1} \mathrm{XL}_{2}(15.65 \mathrm{~mm}), \mathrm{T}_{4} \mathrm{XD}_{2} \mathrm{XL}_{2}(15.47 \mathrm{~mm}), \mathrm{T}_{4} \mathrm{XD}_{3} \mathrm{XL}_{2}(15.31 \mathrm{~mm})$ and $\mathrm{T}_{6} \mathrm{XD}_{3} \mathrm{XL}_{1}(14.35 \mathrm{~mm})$. Again, there was no significant variation among $\mathrm{T}_{6} \mathrm{XD}_{3} \mathrm{XL}_{1}(14.55 \mathrm{~mm}), \mathrm{T}_{1} \mathrm{XD}_{2} \mathrm{XL}_{2}(13.93 \mathrm{~mm}), \mathrm{T}_{2} \mathrm{XD}_{2} \mathrm{XL}_{2}$ (14.18mm), $\mathrm{T}_{3} \mathrm{XD}_{2} \mathrm{XL}_{2}(14.25 \mathrm{~mm}), \mathrm{T}_{1} \mathrm{XD}_{3} \mathrm{XL}_{2}(14.45 \mathrm{~mm}), \mathrm{T}_{2} \mathrm{XD}_{3} \mathrm{XL}_{2}$ $(13.95 \mathrm{~mm})$ and $\mathrm{T}_{3} \mathrm{XD}_{3} \mathrm{XL}_{2}(14.23 \mathrm{~mm})$. The highest result was found in $\mathrm{T}_{6} \mathrm{XD}_{1} \mathrm{XL}_{2}(19.57 \mathrm{~mm})$ and lowest result was found in $\mathrm{T}_{5} \mathrm{XD}_{3} \mathrm{XL}_{2}$ $(13.76 \mathrm{~mm})$.

Interaction effect between locations and different types of containers and seed treatments on fiber yield/ha were found significant. But there was no significant differences among $\mathrm{T}_{1} \mathrm{XD}_{1} \mathrm{XL}_{1}$ (5.46ton), $\mathrm{T}_{2} \mathrm{XD}_{1} \mathrm{XL}_{1}$ (6.81ton), $\mathrm{T}_{5} \mathrm{XD}_{1} \mathrm{XL}_{1}$ (6.21ton) and $\mathrm{T}_{6} \mathrm{XD}_{1} \mathrm{XL}_{1}$ (6.07ton). Again, there was no significant variation among $\mathrm{T}_{4} \mathrm{XD}_{1} \mathrm{XL}_{1}$ (3.69ton), $\mathrm{T}_{1} \mathrm{XD}_{2} \mathrm{XL}_{1}$ (2.66ton), $\mathrm{T}_{2} \mathrm{XD}_{2} \mathrm{XL}_{1}$ (3.36ton), $\mathrm{T}_{3} \mathrm{XD}_{2} \mathrm{XL}_{1}$ (3.59ton), $\mathrm{T}_{4} \mathrm{XD}_{2} \mathrm{XL}_{1}$ (3.17ton), $\mathrm{T}_{5} \mathrm{XD}_{2} \mathrm{XL}_{1}$ (3.69ton), $\mathrm{T}_{1} \mathrm{XD}_{3} \mathrm{XL}_{1}$ (3.08ton), $\mathrm{T}_{2} \mathrm{XD}_{3} \mathrm{XL}_{1}$ (2.33ton), $\mathrm{T}_{3} \mathrm{XD}_{3} \mathrm{XL}_{1}$ (2.19ton), $\mathrm{T}_{4} \mathrm{XD}_{3} \mathrm{XL}_{1}$ (2.80ton), $\mathrm{T}_{5} \mathrm{XD}_{3} \mathrm{XL}_{1}$ (3.08ton), $\mathrm{T}_{6} \mathrm{XD}_{3} \mathrm{XL}_{1}$ (2.66ton), $\mathrm{T}_{1} \mathrm{XD}_{1} \mathrm{XL}_{2}$ (3.55ton), $\mathrm{T}_{2} \mathrm{XD}_{1} \mathrm{XL}_{2}$ (3.06ton), $\mathrm{T}_{4}^{6} \mathrm{XD}_{1} \mathrm{XL}_{2}$ (3.09ton), $\mathrm{T}_{5} \mathrm{XD}_{1} \mathrm{XL}_{2}^{2}$ (3.32ton), $\mathrm{T}_{6} \mathrm{XD}_{1} \mathrm{XL}_{2}$ (3.36ton), $\mathrm{T}_{1} \mathrm{XD}_{2} \mathrm{XL}_{2}$ (3.50ton), $\mathrm{T}_{2} \mathrm{XD}_{2} \mathrm{XL}_{2}$ (2.88ton), 
$\mathrm{T}_{3} \mathrm{XD}_{2} \mathrm{XL}_{2}$ (2.67ton), $\mathrm{T}_{4} \mathrm{XD}_{2} \mathrm{XL}_{2}$ (3.45ton), $\mathrm{T}_{5} \mathrm{XD}_{2} \mathrm{XL}_{2}$ (3.44ton), $\mathrm{T}_{6} \mathrm{XD}_{2} \mathrm{XL}_{2}$ (3.77ton), $\mathrm{T}_{1} \mathrm{XD}_{3} \mathrm{XL}_{2}$ (2.48ton) and $\mathrm{T}_{4} \mathrm{XD}_{3} \mathrm{XL}_{2}$ (3.16ton). The highest result was found in $\mathrm{T}_{2} \mathrm{XD}_{1} \mathrm{XL}_{1}$ (6.81ton) followed by $\mathrm{T}_{5} \mathrm{XD}_{1} \mathrm{XL}_{1}$ (6.21ton). The lowest result was found in $\mathrm{T}_{6} \mathrm{XD}_{3} \mathrm{XL}_{2}$ (1.39ton) preceded by $\mathrm{T}_{5} \mathrm{XD}_{3} \mathrm{XL}_{2}$ (1.90ton).

Interaction effects between locations and different types of containers and seed treatments on stick yield/ha were found significant. But, there was no significant differences among $\mathrm{T}_{1} \mathrm{XD}_{1} \mathrm{XL}_{1}$ (10.76ton), $\mathrm{T}_{2} \mathrm{XD}_{1} \mathrm{XL}_{1}$ (11.14ton) and $\mathrm{T}_{5} \mathrm{XD}_{1} \mathrm{XL}_{1}$ (11.04ton). Again, there was no significant variation among $\mathrm{T}_{4} \mathrm{XD}_{1} \mathrm{XL}_{1}$ (6.88ton), $\mathrm{T}_{3} \mathrm{XD}_{2} \mathrm{XL}_{1}$ (6.91ton), $\mathrm{T}_{4} \mathrm{XD}_{2} \mathrm{XL}_{1}$ (6.62ton), $\mathrm{T}_{5} \mathrm{XD}_{2} \mathrm{XL}_{1}$ (6.44ton), $\mathrm{T}_{4} \mathrm{XD}_{1} \mathrm{XL}_{2}$ (6.88ton), $\mathrm{T}_{5} \mathrm{XD}_{1} \mathrm{XL}_{2}$ (7.04ton), $\mathrm{T}_{3} \mathrm{XD}_{2} \mathrm{XL}_{2}$ (6.91ton), $\mathrm{T}_{4} \mathrm{XD}_{2} \mathrm{XL}_{2}$ (6.62ton) and $\mathrm{T}_{5} \mathrm{XD}_{2} \mathrm{XL}_{2}$ (6.44ton). The highest result was found in $\mathrm{T}_{2} \mathrm{XD}_{1} \mathrm{XL}_{1}$ (11.14ton) followed by $\mathrm{T}_{5} \mathrm{XD}_{1} \mathrm{XL}_{1}$ (11.04ton). The lowest result was found in $\mathrm{T}_{3} \mathrm{XD}_{3} \mathrm{XL}_{2}$ (4.33ton) preceded by $\mathrm{T}_{5} \mathrm{XD}_{3} \mathrm{XL}_{2}$ (4.63ton)

Interaction effects between different types of containers and seed treatments on seed yield/ha were found significant. But, there were no significant differences among $\mathrm{T}_{2} \mathrm{XD}_{1} \mathrm{XL}_{1}(356.32 \mathrm{~kg}), \mathrm{T}_{2} \mathrm{XD}_{2} \mathrm{XL}$ $(356.23 \mathrm{~kg}), \quad \mathrm{T}_{2} \mathrm{XD}_{3} \mathrm{XL}_{1} \quad(314.34 \mathrm{~kg}), \quad \mathrm{T}_{4} \mathrm{XD}_{3} \mathrm{XL}_{1} \quad(313.65 \mathrm{~kg})$ and $\mathrm{T}_{4} \mathrm{XD}_{3} \mathrm{XL}_{2}(313.65 \mathrm{~kg})$. The highest result was found in $\mathrm{T}_{1} \mathrm{XD}_{1} \mathrm{XL}$ $(422.44 \mathrm{~kg})$ followed by $\mathrm{T}_{1} \mathrm{XD}_{1} \mathrm{XL}_{2}(418.55 \mathrm{~kg})$. The lowest result was found in $\mathrm{T}_{3} \mathrm{XD}_{2} \mathrm{XL}_{2}(322.55 \mathrm{~kg})$ preceded by $\mathrm{T}_{4} \mathrm{XD}_{2} \mathrm{XL}_{2}(323.43 \mathrm{~kg})$.

Interaction effects between locations and different types of containers and seed treatments on 1000-seed weight were found significant. But there was no significant differences among $\mathrm{T}_{1} \mathrm{XD}_{1} \mathrm{XL}_{1}$ (1.49gm), $\mathrm{T}_{2} \mathrm{XD}_{1} \mathrm{XL}_{1}(1.67 \mathrm{gm}), \mathrm{T}_{3} \mathrm{XD}_{1} \mathrm{XL}_{1}(1.84 \mathrm{gm}), \mathrm{T}_{5} \mathrm{XD}_{1} \mathrm{XL}_{1}$ (1.56gm), $\mathrm{T}_{1} \mathrm{XD}_{2} \mathrm{XL}_{1}(1.45 \mathrm{gm}), \mathrm{T}_{2} \mathrm{XD}_{2} \mathrm{XL}_{1}(1.75 \mathrm{gm}), \mathrm{T}_{3} \mathrm{XD}_{2} \mathrm{XL}_{1}$ (1.67gm), $\mathrm{T}_{5} \mathrm{XD}_{2} \mathrm{XL}_{1}(1.55 \mathrm{gm}), \mathrm{T}_{1} \mathrm{XD}_{3} \mathrm{XL}_{1}(1.45 \mathrm{gm}), \mathrm{T}_{2} \mathrm{XD}_{3} \mathrm{XL}_{1}$ (1.68gm), $\mathrm{T}_{1} \mathrm{XD}_{1} \mathrm{XL}_{2}(1.39 \mathrm{gm}), \mathrm{T}_{2} \mathrm{XD}_{1} \mathrm{XL}_{2}(1.85 \mathrm{gm}), \mathrm{T}_{3} \mathrm{XD}_{1} \mathrm{XL}_{2}$ (1.78gm), $\mathrm{T}_{5} \mathrm{XD}_{1} \mathrm{XL}_{2}(1.53 \mathrm{gm}), \mathrm{T}_{1} \mathrm{XD}_{2} \mathrm{XL}_{2}(1.32 \mathrm{gm}), \mathrm{T}_{2} \mathrm{XD}_{2} \mathrm{XL}_{2}$ $(1.65 \mathrm{gm}), \mathrm{T}_{4} \mathrm{XD}_{2} \mathrm{XL}_{2}(1.42 \mathrm{gm})$ and $\mathrm{T}_{1} \mathrm{XD}_{3} \mathrm{XL}_{2}(1.39 \mathrm{gm})$. The highest result was found in $\mathrm{T}_{2} \mathrm{XD}_{1} \mathrm{XL}_{1}(1.85 \mathrm{gm})$ followed by $\mathrm{T}_{3} \mathrm{XD}_{1} \mathrm{XL}_{1}$ (1.84gm). The lowest result was found in $\mathrm{T}_{3} \mathrm{XD}_{2} \mathrm{XL}_{2}(1.11 \mathrm{gm})$ preceded by $\mathrm{T}_{5} \mathrm{XD}_{3} \mathrm{XL}_{1}, \mathrm{~T}_{6} \mathrm{XD}_{1} \mathrm{XL}_{2}, \mathrm{~T}_{6} \mathrm{XD}_{2} \mathrm{XL}_{1}$ and $\mathrm{T}_{2} \mathrm{XD}_{3} \mathrm{XL}_{2}$ (1.12gm) (Table 4).

\section{Discussion}

The experiments were conducted in the field of Jute Agriculture Experimental Station (JAES), Manikgonj and Kishoregonj Regional Station (KRS), Kishoregonj of BJRI. Six different types of containers viz., tin pot, plastic pot, poly bag, gunny bag lined with polyethylene, cloth bag, and the IRRI poly bag, two different seed treatments viz. Provax-200 and B and two locations viz. JAES and KRS were used for the present study. Effects of seed treatments with B and Provax-200 on the production of quality healthy seeds were studied. Disease incidence occurred minimum at JAES and KRS when seeds were treated with B. Be and Provax-200 have been recorded as the superior means for controlling seed borne fungi as well as field fungi with higher seed yield and better improvement of seed quality as similarly reported by Hossain, ${ }^{21,22}$ Hossain et al. ${ }^{23}$ Mostafa, ${ }^{24}$ Hossain and Sarker $^{25}$ Islam and Biswas, ${ }^{26}$ Khan and Fakir ${ }^{27}$ and Haque et al. ${ }^{28}$ The present findings revealed that seed quality was comparatively better in case of using $\mathrm{B}$ and $\mathrm{P}$ treated seeds as well as seed borne infection of fungal pathogens were less in seeds produced by B and $\mathrm{P}$ treated seeds. Moreover, seed treatments increased germination with the decrease of total seed borne fungal pathogens. Similarly,
Akanda and Fakir $^{29}$ recorded low germination of jute seeds having high seed borne fungal pathogens. Hossain $(2011 \mathrm{a})^{30}$ reported that B (3\%) was found to control the seed borne pathogens and also increased the germination percentage of seeds. A similar result was also reported by Yeasmin et al. ${ }^{31}$, Shultana et al., ${ }^{32}$ and Bhuiyan et al. ${ }^{33}$ Haque et al. ${ }^{28}$ reported that seed germination varied significantly with respect to variety, seed category and location of seed collection. The highest disease incidence $(10.86 \%)$ was encountered in case of interaction among plastic pot storing seeds and control condition. Interaction between different types of containers and seed treatments, the highest germination $(68.17 \%$ ) was recorded in interaction effect of tin pot storing seeds and B treated seeds. The stick yield/ha (11.14ton) and fiber yield/ha (6.81ton) were recorded in interaction effect of plastic pot storing seeds and B treated seeds. Highest seed yield/ ha $(422.44 \mathrm{~kg})$ was observed in the interaction of tin pot storing seeds and B treated seeds. Interaction between different types of containers, seed treatments and locations, the highest disease incidence (12.84\%) was encountered in interaction effect of plastic pot storing seeds, control condition and KRS. Highest fiber yield/ha (6.81ton) and stick yield/ha (11.14ton) were recorded in interaction of plastic pot storing seeds, B treated seeds and JAES. Highest seed yield/ ha $(422.44 \mathrm{~kg})$ was recorded in interaction of seed storing in a tin pot, treated with $B$ and JAES location.

\section{Conclusion}

Therefore, the following conclusion may be drawn for quality seed and fiber production, from the findings of this study:

A. B (3\% of seed weight) can successfully be used for treating seeds to avoid $\mathrm{P}$ for the production of quality healthy jute seeds with higher seed and fiber yield.

B. Fiber and seed yield were found to decrease with the increase of seed borne infection of fungal pathogens.

C. Seed germination and disease incidence varied significantly with respect to storage container, seed treatment and location.

So, the following recommendation may be drawn for quality seed and fiber production, from the findings of this study:

a) Quality of jute seeds can be maintained by storage in tin pot and seed treated of B enhance the quality and yield of the jute seed and fiber in the field.

\section{Acknowledgments}

None.

\section{Conflicts of interest}

Authors declare that there is no conflict of interest.

\section{References}

1. Islam MM. About jute seed research. R S printing press: Kalwalapara, Mirpur-1, Dhaka; 2007.

2. BBS. Statistical Year Book of Bangladesh. Bangladesh Bureau of Statistics, Planning Ministry: Dhaka; 2011.

3. www.jute.org

4. Khandakar AL. Jute seed at farm level. Bangladesh Agric Res Coun: Dhaka; 1987. 1-77 p. 
5. Hossain MA, Talukder FAH, Islam M, et al. Studies on C. olitorius pipeline varieties. Ann Rep Bangladesh Jute Res Inst: Dhaka; 1994. 132-137 p.

6. Fakir GA. An annotated list of seed borne diseases in Bangladesh. Seed Pathology Laboratory, Department of Plant Pathology: Mymensingh; 2001. 7-8 p

7. Fakir GA, Islam MR. Survey on the health status of jute and rice seeds of farmers of Sadar Upazilla, Mymensingh. BAURES progress. 1990;4:42-47.

8. Ikata S, Yoshida M. A new anthracnose of jute plant. Annuals of Phytopath Japan. 1940;10:141-149.

9. Fazli SFI, Ahmed QA. Fungus organisms associated with jute seeds and their effect on germinating seeds and seedlings. Agric Pakistan. 1960;11:298-306

10. Ahmed QA. Problems in jute plant pathology. Jute and Jute fabrics Pakistan; 1966. 184-186 p.

11. Fakir GA, Sarder MA, Gaffar A, et al. An annotated list of important disorders of important crop plants of Bangladesh Pl Quarantine Rev Prog Sponsored by the Ministry of Agriculture in coordination with CIDA and DANIDA; 1991. 107 p.

12. Ahmed QA. Diseases of jute in East Pakistan. Jute and Jute Fab Pak. 1968;7:147-151.

13. Ahmed N, Islam N. Correlation between jute seed infections recorded in the laboratory with the incidence diseases in the field. Ann Rep BJRI; 1980. 129-130 p.

14. Biswas AC, Taher MA, Asaduzzaman M, et al. Loss of yield and quality of jute fiber due to prevalence of stem rot. Bangladesh J Plant Pathol. 1985;1(1):61-62.

15. Ahmed N, Sultana K. Survey on the production and quality of jute seeds at Farm level. Ann Rep BJRI: Dhaka; 1985. 296-323 p.

16. Fakir GA. Seed health test in seed quality control and seed certification. Seed Path Lab Pub. 1989;4:11.

17. Anonymous. Control of insects and diseases of jute. Bangladesh Jute Res Inst: Dhaka; 2001. 1-13 p.

18. Islam MM. Jute seed technology. College gate binding and printing: Dhaka; 2009. 89-97 p.

19. Islam MM, Rahman M. Hand book on Agricultural technology of jute, kenaf and mesta crops. Bulbul printers: Dhaka; 2008. 27-28 p.
20. Gomez AK, Gomez AA. Statistical Procedures for Agriculture Research. 2nd ed. Rice Research Institute: Manila, Philippines; 1984. 680 p.

21. Hossain I. Troymashik Krishi Projuktii Barta. BAU Res. 2011;3(8):13-15.

22. Hossain I. Environment friendly control of foot and root rot of lentil and chickpea in the field. BAU Res Prog. 2010;20:29.

23. Hossain I, Yeasmin R, Hossain MM. Management of seedling diseases of blackgram, mungben and lentil using BAU- Biofungicide, biofertilizer and cowdung. Eco-friendly Agril J. 2009;2(11):905-910.

24. Mostafa G. Effect of BAU-Biofungicide in comparison with Bavistin and Amistar for controlling diseases of soybean variety G2. An M. S. Thesis submitted to the Department of Plant Pathology, Bangladesh Agricultural University: Mymensingh; 2009.

25. Hossain I, Sarkar SR. Nursery diseases of mango and their management. Proc Bangladesh Agricultural University Research Progress. 2008;19:37-38.

26. Islam N, Biswas AC. Screening of seed dressing fungicides. Annual Report. BJRI; 1981. 218-219 p.

27. Khan AA, Fakir GA. Association of seed borne pathogens with growing capsules and their entry into developing seeds in jute. Bangladesh $J$ Plant Pathol. 1993;9(1\&2):1-3.

28. Haque MM, Sultana K, Fakir GA. Prevalence of major fungal pathogen in breeder seed, foundation seed, certified seed and farmers'seed of jute. Collaborative Research Report (BJRI and SPL); 1999. 11-16 p.

29. Akanda MAM, Fakir GA. Effect of seed dressing chemicals for the control of major seed borne pathogens of jute. Bangladesh J Pl Pathol. 1985;1(11):13-19.

30. Hossain I. BAU- Bio fungicide: Unique eco-friendly means and new dimension of plant disease control in Bangladesh. Lima Printing Press: Mymensingh; 2011.

31. Yeasmin R, Hossain I, Hossain MM. Management of seedling diseases of blackgram, mungbean and lentil using BAU- Biofungicide, biofertilizer and cowdung. Eco-friendly Agril J. 2009;2(11):905-910.

32. Shultana R, Hossain I, Ahmed S, et al. Efficacy of BAU- Biofungicide in controlling leaf spot of wheat (Triticum aestivum). Eco-friendly Agril J. 2009;2(2):392-395.

33. Bhuiyan MAHB, Khokon MAR, Hossain I. BAU- Biofungicide in controlling seedling diseases of winter vegetables. Bangladesh J Plant Pathol. 2006;22(1\&2):1-5. 